\title{
Successful Treatment of Low-Dose Lenalidomide Maintenance Therapy Followed by Second Autologous Peripheral Blood Stem Cell Transplantation in Heavily Treated Multiple Myeloma
}

\author{
Naohiro Sekiguchi ${ }^{1 *}$, Naoki Takezako ${ }^{1}$, Takashi Ishii ${ }^{1}$, Akihisa Nagata ${ }^{1}$, Satoshi Noto $^{1}$, Akiyoshi Miwa $^{2}$ \\ ${ }^{1}$ Hematology Division, National Hospital Organization Disaster Medical Center, Tokyo, Japan; ${ }^{2}$ Hematology Division, National \\ Center for Global Health and Medicine, Tokyo, Japan. \\ Email: *nao26@aol.com
}

Received December $9^{\text {th }}, 2011$; revised January $24^{\text {th }}, 2012$; accepted February $17^{\text {th }}, 2012$

\begin{abstract}
Recently, the prognosis of multiple myeloma has been improved by using high-dose chemotherapy followed by autologous peripheral blood stem cell transplantation (ASCT), bortezomib, and immunomodulatory drugs including thalidomide and lenalidomide. On the other hand, treatment strategy remains difficult for refractory and relapse cases. Here, we report the successful treatment of low-dose lenalidomide maintenance therapy followed by salvage ASCT in a heavily treated patient with multiple myeloma. This 58-year-old woman with IgG- $\lambda$ multiple myeloma had a 5th recurrence in June, 2011. It was 7 years post-diagnosis, and she had received conventional therapies such as VAD, MP therapy. Furthermore, the patient had already been treated with ASCT, bortezomib, and thalidomide therapy. At the 5th recurrence, she had extramedullary plasmacytoma in the left orbit. She initially received bortezomib and dexamethasone therapy as induction therapy. After peripheral blood stem cell collection, radiation therapy was performed. The patient then received a second ASCT. Three months later, the response was very good partial response. Finally, the patient was treated with $5 \mathrm{mg} / \mathrm{day}$ lenalidomide orally as a maintenance therapy, and she achieved stringent complete response after 2 months according to International Myeloma Working Group response criteria. Low-dose lenalidomide maintenance therapy might be also useful for ASCT as salvage therapy, although further studies are warranted.
\end{abstract}

Keywords: Multiple Myeloma; Stem Cell Transplantation; Lenalidomide; Maintenance Therapy

\section{Introduction}

Recently, myeloma survival has markedly improved by high-dose chemotherapy with autologous peripheral blood stem cell transplantation (ASCT) in young patients [1,2]. Furthermore, novel agents such as bortezomib, and immunomodulatory drugs have been recently become available [2]. On the other hand, this treatment strategy might be considered difficult for refractory and relapse patients. One of the choices of salvage therapy is ASCT, which has been reported useful and safe [3-5]. The other option might involve using novel agents. Some reports are available using novel agents after relapse or progression after frontline therapy including ASCT [6,7]. We report here a case of successful treatment of heavily treated myeloma patient with low-dose lenalidomide maintenance therapy after second ASCT as salvage therapy.

\footnotetext{
${ }^{*}$ Corresponding author.
}

\section{Case Report}

A 58-year-old woman was diagnosed as having 5th recurrence because of the extra medullary mass in June 2011. She was diagnosed with symptomatic multiple myeloma IgG-lambda [8] and clinical stage was II according to the International Scoring System (ISS) [9] in March 2003. She had already received conventional chemotherapy such as VAD [10], MP [11] therapies. Moreover, she was treated with a high-dose of melphalan therapy followed by autologous peripheral blood stem cell transplantation (ASCT), thalidomide, and bortezomib (Table 1). Magnetic resonance imaging also showed an extra medullary mass of the left orbital part of the frontal bone (Figure 1(a)). At the 5th recurrence, anemia, renal dysfunction, and hypercalcemia were not detected. Serum protein electrophoresis showed an M-spike, and IgG was $2284 \mathrm{mg} / \mathrm{dL}$ (normal range: 870 - 1700), while IgA and IgM were suppressed, $38 \mathrm{mg} / \mathrm{dL}$, and $42 \mathrm{mg} / \mathrm{dL}$, respectively. Bence-Jones protein was positive in the urine. Bone marrow aspirate 
Table 1. Therapy history.

\begin{tabular}{llll}
\hline Time & Status & Therapy & Response \\
\hline Sept. 2003 & at diagnosis & VAD, ASCT & PR \\
Oct. 2005 & $1^{\text {st }}$ recurrence & TD & SD \\
Oct. 2006 & $2^{\text {nd }}$ recurrence & HD-Dex & SD \\
Nov. 2007 & $3^{\text {rd }}$ recurrence & MPT & SD \\
Apr. 2008 & $4^{\text {th }}$ recurrence & BD & VGPR \\
Jun. 2010 & $5^{\text {th }}$ Recurrence & &
\end{tabular}

VAD: vincristine, adriamycin, dexamethason; ASCT: autologous peripheral blood stem cell transplantation; TD: thalidomide, dexamethasone; MPT: melphalan, prednisolone, thalidomide; BD: bortezomib, dexamethasone; PR: partial response; SD: stable disease; VGPR: very good partial response.

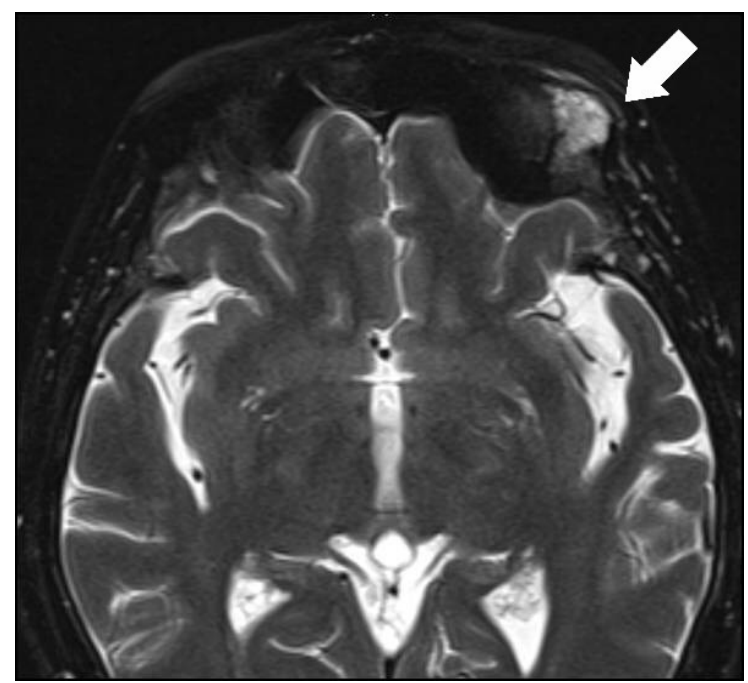

(a)

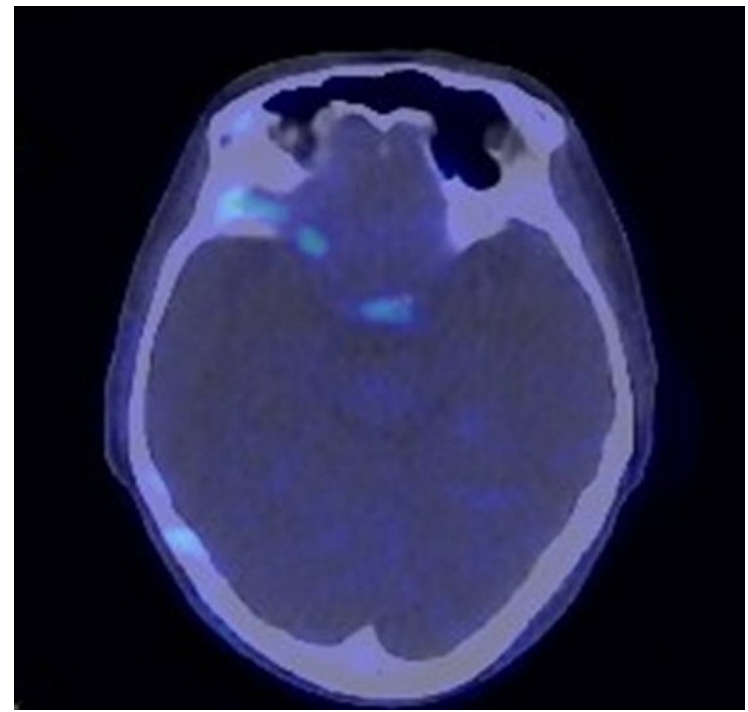

(b)

Figure 1. (a) Magnetic resonance imaging showed the extra medullary mass had high signal intensity (Arrow); (b) [18F]-fluorodeoxyglucose Positoron Emittion tomography scan showed the plasmacytoma disappeared. revealed diffuse infiltration of myeloma cells. Conventional cytogenetic analysis showed normal karyotype, on the other hand, fluorescence in-situ hybridization (FISH) analysis revealed positive for del13q, IgH-FGFR3, and negative for IgH-Bcl-1, IgH-C-MYC, and del17p13. Her performance status was 1 by Eastern Cooperative Oncology Group criteria.

We first performed 1cycle of bortezomib and dexamethasone therapy. As the patient experienced grade 2 peripheral neuropathy (PN), we discontinued the therapy. Next, we tried peripheral stem cell collection after etoposide, dexamethasone, cytarabine, and cisplatin therapy [12], followed by radiation therapy of 40 Gy divided into 20 fractions. The PN had been recovered into grade 1 after the radiation therapy. After that, the patient received ASCT. The conditioning regimen [13] consisted of bortezomib in addition to high dose melphalan. Bortezomib was administered intravenously at $1 \mathrm{mg} / \mathrm{m}^{2}$ on days -6 , $-3,1$, and 4, with dexamethasone $16 \mathrm{mg} /$ body. Melphalan was administered intravenously at $200 \mathrm{mg} / \mathrm{m}^{2}$ on day -2 . The response after three months of the ASCT was very good partial response (VGPR) according to the International Myeloma Working Group (IMWG) response criteria [14]. We then administered $5 \mathrm{mg}$ of lenalidomide orally as maintenance therapy. After 2 months of maintenance therapy, the serum free light chain kappa/lambda ratio was 0.67 (normal range: 0.26 - 1.65) and flow cytometric analysis of the kappa/lambda ratio of neoplastic cells was normal by bone marrow aspirate. [18F]-fluorodeoxyglucose positron emission tomography-computed tomography showed no extramedullary mass (Figure 1(b)). Therefore, she achieved stringent complete response (sCR) according to the IMWG response criteria. sCR has since been maintained for 6 months.

\section{Discussion}

Recently, myeloma survival has been markedly improved by ASCT [1], bortezomib, and immunomodulatory drugs including thalidomide and lenalidomide [1,2]. Furthermore, the prolonged survival has been reported for patients with recurrence after ASCT using novel agents [6,7]. It was also reported that second transplantation was effective and safe as salvage therapy [3-5]. However, more than 4 prior-lines of therapies are reported as prognostic factors for worsening after second ASCT [3]. She had already received 5 prior-line therapies, and had been administered novel agents such as thalidomide and bortezomib, thus, a limited effect might have been expected from the second ASCT.

Recently, much data concerned with conventional cytogenetic and FISH analysis has been accumulated and which might be widely considered related to the prognosis $[15,16]$. In the present case, del13q and IgH-FGFR3 
were recognized by FISH analysis. IgH-FGFR3 rearrangement was generally considered as a worse prognostic factor of high dose melphalan therapy [15]. On the other hand, it was reported that bortezomib containing regimens might be effective in patients with IgH-FGFR3 rearrangements [17]. Indeed, the patient in the present case achieved VGPR with bortezomib and dexamethasone therapy at the 4th recurrence. Thus, we administered bortezomib in addition to high-dose melphalan as conditioning regimen [13], as has been done by a French group [18].

The impact of maintenance therapies has also been reported [19-22]. Very recently, McCarthy et al. reported the superior overall survival of low-dose lenalidomide therapy as maintenance therapy after the first-line ASCT compared to placebo in a phase III intergroup study [22]. We administered low-dose lenalidomide as a maintenance therapy after second transplantation. Lenalidomide has been reported to have multiple mechanisms in the treatment of myeloma patients. With the low-dose of lenalidomide therapy, the mechanism for anti-myeloma effect might be mainly immunomodulatory effects and anti-inflammatory activities, while it might not have direct antitumor activities in this setting, and anti-angiogenesis from in vitro studies and pharmacokinetics studies in vivo [2325]. Our patient achieved sCR after two months of low dose lenalidomide maintenance therapy.

It was already 7 years after diagnosis, and she had been a heavily treated patient. Despite this the present case achieved sCR with low-dose lenalidomide as maintenance therapy after second ASCT. In conclusion, lowdose lenalidomide maintenance therapy might be useful for ASCT as salvage therapy, although further studies are warranted.

\section{REFERENCES}

[1] J. Koreth, C. S. Cutler and B. Djurvegovic, "High-Dose Therapy with Single Autologous Transplantation versus Chemotherapy for Newly Diagnosed Multiple Myeloma: A Systematic Review and meta-analysis of Randomized Controlled Trials," Biology of Blood and Marrow Transplantation, Vol. 13, No. 2, 2007, pp. 183-196. doi:10.1016/j.bbmt.2006.09.010

[2] I. Turesson, R. Velez and S. Y. Kristinsson, "Patterns of Improved Survival in Patients with Multiple Myeloma in the Twenty-First Century: A Population-Based Study," Journal of Clinical Oncology, Vol. 28, No. 5, 2010, pp. 830-834. doi:10.1200/JCO.2009.25.4177

[3] R. L. Olin, D. T. Vogl and D. L. Porter, "Second AutoSCT Is Safe and Effective Salvage Therapy for Relapsed Multiple Myeloma,” Bone Marrow Transplantation, Vol. 43, No. 5, 2009, pp. 417-422. doi:10.1038/bmt.2008.334

[4] R. Fenk, V. Liese and F. Neubauer, "Predictive Factors for Successful Salvage High-Dose Therapy in Patients with Multiple Myeloma Relapsing after Autologous Blood
Stem Cell Transplantation," Leukemia and Lymphoma, Vol. 52, 2011, pp. 1455-1462. doi:10.3109/10428194.2011.575967

[5] F. Elice, R. Raimondi and A. Tosetto, "Prolonged Overall Survival with Second On-Demand Autologous Transplant in Multiple Myeloma,” American Journal of Hematology, Vol. 81, No. 6, 2006, pp. 426-431. doi:10.1002/ajh.20641

[6] S. K. Kumar, S. V. Rajkumar and A. Dispenzieri, "Improved Survival in Multiple Myeloma and the Impact of Novel Therapies," Blood, Vol. 111, No. 5, 2008, pp. 2516-2520. doi:10.1182/blood-2007-10-116129

[7] A. Palumbo, P. Falco and M. T. Ambrosini, "Thalidomide plus Dexamethasone Is an Effective Salvage Regimen for Myeloma Patients Relapsing after Autologous Transplant,” European Journal of Haematology, Vol. 75, No. 5, 2005, pp. 391-395. doi:10.1111/j.1600-0609.2005.00533.x

[8] International Myeloma Working Group, "Criteria for the Classification of Monoclonal Gammopathies, Multiple Myeloma and Related Disorders: A Report of the International Myeloma Working Group," British Journal of Haematology, Vol. 121, No. 5, 2003, pp. 749-757. doi:10.1046/j.1365-2141.2003.04355.x

[9] P. R. Greipp, J. San Miguel and B. G. M. Durie, "International Staging System for Multiple Myeloma," Journal of Clinical Oncology, Vol. 23, No. 15, 2005, pp. 3412-3420. doi:10.1200/JCO.2005.04.242

[10] B. Barlogie, L. Smith, and R. Alexanian, "Effective Treatment of Advanced Multiple Myeloma Refractory to Alkylating Agent," New England Journal of Medicine, Vol. 310, No. 21, 1984, pp.1353-1356. doi:10.1056/NEJM198405243102104

[11] A. Raymond, R. Alexanian and A. Haut, "Combination Chemotherapy with Different Melphalan Dose Regimens," Journal of American Medical Association, Vol. 208, No. 9, 1969, pp. 1680-1685. doi:10.1001/jama.1969.03160090040009

[12] B. Barlogie, W. S. Velasquez and R. Alexanian, "Etoposide, Dexamethasone, Cytarabine, and Cisplatin in Vincristine, Doxorubicin, and Dexamethasone-Refractory Myeloma,” Journal of Clinical Oncology, Vol. 7, No. 10, 1989, pp. 1514-1517.

[13] N. Takezako, N. Sekiguchi and A. Nagata, “Conditioning for Autologous Stem Cell Transplantation by Combining Bortezomib and Dexamethasone with High-Dose Melphalan (BD-HDM) Is Feasible in Young Japanese Multiple Myeloma Patients," Hematologica, Vol.96, No. 1, 2011, p. 93.

[14] B. G. Durie, J. L. Haroussaeu and J. S. Miguel, "International Uniform Response Criteria for Multiple Myeloma,” Leukemia, Vol. 20, No. 9, 2006, pp. 1467-1473. doi:10.1038/sj.leu.2404284

[15] R. Fonseca, P. L. Bergsagel and J. Drach, "International Myeloma Working Group Molecular Classification of Multiple Myeloma: Spotlight Review," Leukemia, Vol. 23, No. 12, 2009, pp. 2210-2221. doi:10.1038/leu.2009.174 
[16] S. K. Kumar, J. R. Mikhael and F. K. Buadi, "Management of Newly Diagnosed Symptomatic Multiple Myeloma: updated Mayo Stratification of Myeloma and RiskAdapted Therapy (mSMART) Consensus Guidelines,” Mayo Clinic Proceedings, Vol. 84, No. 12, 2009, pp. 1095-1110. doi:10.4065/mcp.2009.0603

[17] J. F. San Miguel, R. Schlag and N. K. Khuageva, "Bortezomib Plus Melphalan and Prednisone for Initial Treatment of Multiple Myeloma," New England Journal of Medicine, Vol. 359, No. 9, 2008, pp. 906-917. doi:10.1056/NEJMoa0801479

[18] M. Roussel, P. Moreau and A. Huynh, "Bortezomib and High-Dose Melphalan as Conditioning Regimen before Autologous Stem Cell Transplantation in Patients with de Novo Multiple Myeloma: A Phase 2 Study of the Intergroupe Francophone du Myelome (IFM)," Blood, Vol. 115 , No. 1, 2010, pp. 32-37. doi:10.1182/blood-2009-06-229658

[19] L. K. Hicks, A. E. Haynes and D. E. Reece, "A MetaAnalysis and Systematic Review of Thalidomide for Patients with Previously Untreated Multiple Myeloma," Cancer Treatment Reviews, Vol. 34, No. 5, 2008, pp. 442-452. doi:10.1016/j.ctrv.2008.02.003

[20] A. Z. Badros, "The Role of Maintenance Therapy in the Treatment of Multiple Myeloma,” Journal of the National Comprehensive Cancer Network, Vol. 8, Suppl. 1, 2010, pp. S21-S27.

[21] G. J. Morgan, W. M. Gregory and F. E. Davies, "The Role of Maintenance Thalidomide Therapy in Multiple Myeloma: MRC Myeloma IX Results and Meta-Analysis," Blood, 2011, in Press.

[22] P. McCarthy, K. Owzar and K. Anderson, "A Phase III Randomized, Double-Blind Study of Maintenance Therapy with Lenalidomide (CC 5013) or Placebo Following Autologous Stem Cell Transplantation for Multiple Myeloma. CALGB, ECOG and BMT CTN 100104,” Hematologica, Vol. 96, No. 1, 2011, p. 23.

[23] T. Hideshima, D. Chauhan and Y. Shima, "Thalidomide and Its Analogs Overcome Drug Resistance of Human Multiple Myeloma Cells to Conventional Therapy,” Blood, Vol. 96, No. 9, 2000, pp. 2943-2950.

[24] L. G. Corral, P. A. Haslett and G. W. Muller, "Differential Cytokine Modulation and T Cell Activation by Two Distinct Classes of Thalidomide Analogues That Are Potent Inhibitors of TNF-Alpha," Journal of Immunology, Vol. 163, No. 1, 1999, pp. 380-386.

[25] L. Lu, F. Payvandi and L. Wu, "The Anti-Cancer Drug Lenalidomide Inhibits Angiogenesis and Metastasis via Multiple Inhibitory Effects on Endothelial Cell Function in Normoxic and Hypoxic Conditions," Microvascular Research, Vol. 77, No. 2, 2009, pp. 78-86. doi:10.1016/j.mvr.2008.08.003 